\title{
Introduction: education and fertility in low-fertility settings
}

\section{Tomáš Sobotka, Éva Beaujouan and Jan Van Bavel*}

This issue of the Vienna Yearbook of Population Research (2017) gives a variety of perspectives on the linkages between education and fertility in low-fertility settings. It follows up on earlier volumes of the Vienna Yearbook that have been devoted to education and demography (2010) and to education and the global fertility transition (2012).

In countries with highly educated populations, educational enrolment and attainment continue to be strongly related to fertility timing, family size, union formation, partnership choices, and broader family behaviours. Cleland (2009: 183) has suggested that the education of adults "persistently emerges as the single most powerful predictor of their demographic behaviour". In turn, fertility and partnership-related preferences can simultaneously shape educational and family trajectories (Stange 2011). The studies in this volume confirm the continuing salience of education for patterns of fertility, family, and reproduction. James et al. (2012: 2) have argued that education "is likely to have increased in importance as a status-defining characteristic over the 20th century". At the same time, the influence of education is moulded by the institutional contexts of different societies, including by each country's educational system, family policies, economic development level, labour market characteristics, family patterns, gender norms, and prevailing societal values.

In recent decades, rich countries with low levels of fertility have been changing substantially, and the opportunities, challenges, and responses created by these transformations have been differentiated along educational lines. Access to higher education has been expanding rapidly, especially among women. In some of these countries - including Canada, Japan, South Korea, and the United Kingdom - a majority of young people are earning tertiary degrees (OECD 2017a).

\footnotetext{
* Tomáš Sobotka (corresponding author), Wittgenstein Centre for Demography and Global Human Capital, Vienna Institute of Demography, Welthandelsplatz 2, Level 2, 1020, Vienna, Austria Email: tomas.sobotka@oeaw.ac.at

Éva Beaujouan, Wittgenstein Centre for Demography and Global Human Capital, Vienna Institute of Demography, Vienna, Austria

Jan Van Bavel, Centre for Sociological Research, University of Leuven, Belgium
} 
As young adults often spend their early to mid-twenties enrolled in education and economically inactive, they are postponing their transitions to employment, residential independence, union formation, and parenthood. A number of studies have demonstrated that educational expansion has been the most prominent factor driving the shift towards later family formation (e.g. Neels et al. 2017; Ní Bhrolcháin and Beaujouan 2012; Mills et al. 2011).

Labour markets have changed rapidly as well. They have become more competitive, both nationally, with individual qualification and work flexibility requirements rising; and internationally, with countries and regions engaged in a global competition for jobs and investments. Combined with labour market deregulation, this competition has often resulted in rising levels of economic uncertainty and the deepening of divides in skills, incomes, and economic opportunities across population groups. As a result of these trends, young adults are more likely than their older counterparts to end up in unstable jobs with relatively low pay and low levels of protection (Mills and Blossfeld 2013). As income inequality has been rising in many countries since the 1980s, social status differences have also been widening (Piketty and Zucman 2014; review by Adserà in this volume). The trends towards more competitive labour markets, lower levels of protection and pay for younger workers, and higher levels of income inequality were accelerated during the economic recession in 2008-13. The economic downturn has negatively affected the long-term prospects for employment, wages, and career (OECD 2014), especially among the Millennials born in the 1980s and early 1990s. Thus, the social status inequalities have grown rapidly in recent years, leaving younger people - and especially those with low to medium levels of education - worse off than the previous generations (e.g. review by Adserà in this volume). These new educational and generational divides are reflected in divergent patterns of union formation and parenthood among women and men with different levels of education. A pattern of disadvantage characterised by early and unstable family transitions has been observed among less educated people (McLanahan and Percheski 2008; Perelli-Harris and Lyons Amos 2016). However, the effects of the economic shocks of the recent recession differed widely across countries, with some countries - especially in Southern Europe - experiencing very high levels of unemployment and long-lasting negative effects on the well-being and the economic position of the younger generations (OECD 2014, 2016).

In parallel, rising gender equality has improved the position of women in the labour market, but also in the private domain, as childcare and household responsibilities are increasingly being shared by men. Women in most rich societies have achieved high levels of labour force participation, and having two incomes has become the norm among couples. However, this general trend has been developing very unevenly across countries, and many forms of gender inequality persist even in the most gender-equal societies, including differences between men and women in rates of part-time work, working hours, and pay (OECD 2017b). Moreover, many working adults continue to struggle to reconcile their career and family aspirations. Gender equality has emerged as one of the key factors influencing the relationship 
between education and family behaviour. Esping-Andersen and Billari (2015) have argued that in gender-egalitarian societies, there has been a trend among highly educated women towards "more family", or towards higher fertility, more frequent marriage, and higher levels of union stability. In parallel, less educated women have experienced lower levels of union stability and, possibly, lower fertility. Similarly, Goldscheider et al. (2015) have highlighted the role of rising education in reducing gender inequalities and in spreading egalitarian gender attitudes over the course of the "gender revolution". This shift has in turn been contributing to the reversals in educational gradients in family behaviours, especially in rates of divorce and union dissolution. Today, many highly educated women earn more than their male partners. This trend has been accompanied by an increase in homogamy and hypogamy in union formation (i.e. the male partner has a lower or a similar educational level) (Klesment and Van Bavel 2017), which is likely shifting the pillars and the processes of reproductive decision-making (Van Bavel 2012).

Clearly, the influence of education on fertility is shaped by a wide array of forces and trends that differ between countries, welfare state regimes, and broad regions; but also between sub-populations. At the same time, this influence is not unidirectional: decisions about education, partnership, and childbearing are often interrelated, and family and partnership-related preferences also shape educational trajectories (Martín-García 2008; Nisén 2016). For instance, women who eventually become mothers have been shown to reduce their investment in education well before the occurrence of parenthood or pregnancy (Stange 2011).

The contributions in this volume document well the context-specific nature of the link between education and family behaviours, especially with respect to fertility levels, fertility timing, childlessness, non-marital childbearing, and fertility intentions. Before reviewing the individual articles, we highlight broader links between them, as summarised in Table 1. Besides their common focus on education several features stand out among the eight published research articles (not counting the debate contributions and the review by Adserà):

- Five studies (by Merz and Liefbroer, Lakomý, Trimarchi and Van Bavel, Oppermann, and Testa and Stephany) apply a comparative perspective that gives their findings broader relevance than those of single-country studies. The first two studies (Merz and Liefbroer, Lakomý) compare the completed fertility levels of larger groups of countries.

- Six studies go beyond the usual focus on women's fertility by analysing the fertility or the fertility intentions of both women and men (Merz and Liefbroer, Lakomý, Testa and Stephany), or by adopting a couple perspective to study fertility and childbearing outside marriage (Trimarchi and Van Bavel, PeriRotem, Bagavos).

- Six studies (Merz and Liefbroer, Lakomý, Trimarchi and Van Bavel, Bagavos, Oppermann, Dubuc) apply a cohort perspective that allows the authors to investigate the quantum and the timing of fertility without having to deal with the tempo effect, which can distort period indicators. 
- Four studies provide a more detailed look at family size distributions (Oppermann, Dubuc), at parity-specific patterns of family building (Bagavos), and at reproductive intentions (Testa and Stephany). Both Oppermann and Dubuc also explore childlessness, while the two studies dealing with nonmarital childbearing (Peri Rotem, Trimarchi and Van Bavel) focus on union status prior to and at the first birth.

- Five studies focus on fertility levels (Merz and Liefbroer, Lakomý, Bagavos, Oppermann, Dubuc). Two of these studies also look at the timing of childbearing (Bagavos, Dubuc).

In the following sections, we outline the contents of the contributions in this volume. We first provide highlights from the invited review and the research articles. Then we explore the key messages and arguments of the contributions in the "demographic debate" section. We conclude by discussing selected findings and proposing an agenda for future research.

\section{Education-fertility links in context: the role of labour market, policies, welfare systems, attitudes, and family status}

The review article by Alícia Adserà provides a broad perspective on changing labour market conditions, rising inequality, and differences in fertility by educational level across rich, low-fertility countries. In particular, Adserà discusses the effects of structural changes in the labour market, including widening income disparities and "disappearing" middle-income jobs. This job polarisation is likely to place downward pressure on the fertility of medium educated women. Adserà also highlights the continuing salience of gender pay gaps and the selection of women into lower-paid jobs (especially in the public sector) that are more secure and more flexible, and thus enable women to combine employment with raising children, but that also hinder their career prospects. She notes that despite these barriers, highly educated women are arguably in a better position to realise their fertility plans now than they were in the past. The better career prospects and higher income levels of highly educated women, coupled with their rising levels of partnership homogamy, imply greater chances of having a stable partnership. Thus, "increases in fertility among women at the top of the educational distribution" are anticipated. In contrast, women and men with lower levels of education are more likely to have unstable partnerships and limited resources, which may be expected to depress their fertility rates. These trends will lead to either a flattening of the educational gradient in fertility or the emergence of a U-shaped pattern.

Two contributions, by Eva-Maria Merz and Aart C. Liefbroer and by Martin Lakomý, analyse completed fertility by level of education among men and women in broader groups of countries, controlling for selected individual characteristics. Merz and Liefbroer use data from the European Social Survey (ESS), and refine the widely used welfare state typology by Esping-Andersen to structure their 


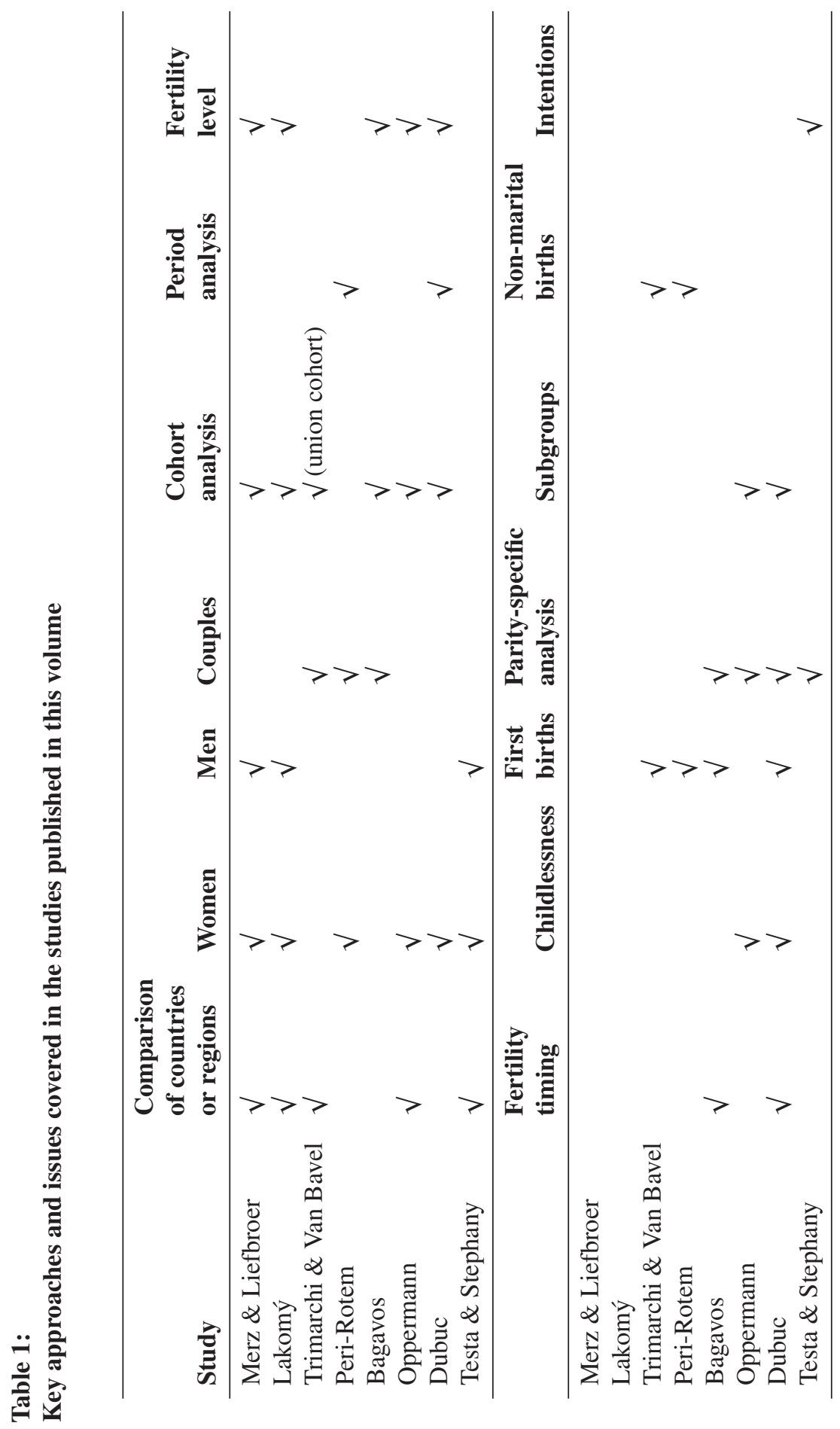


findings. They report a negative educational gradient in fertility for both sexes, which is, however, weaker among men and among more recent cohorts born since 1945. Among women, the educational gradient has weakened more in Western European countries with a "conservative corporative" welfare regime. This trend may be the result of family policies that encourage the reconciliation of work and parenthood, especially in Belgium and France. In contrast, the strongest and most stable negative educational gradient in fertility among women is found in postcommunist countries in Central and Eastern Europe (except for the former Soviet Union). Such patterns are especially interesting given the official egalitarianism and limited income differences between social groups that characterised these countries during the state-socialist era (i.e. until the 1980s). Lakomý's study also finds that there is a negative education-fertility gradient among women across broader regions in Europe. Among men, the impact of education is much weaker and varies by region. The paper contrasts two broad perspectives on fertility: the rational choice framework and the second demographic transition perspective, which stresses the importance of values focused on higher-order needs, self-realisation, and selffulfilment. To gain insight into the possible role of values, the author uses data from the European Values Survey (EVS), which contains a battery of questions on values and attitudes towards marriage, reproduction, family, and family relations. The analysis shows that higher socio-economic status (education and occupation) as well as having more "liberal" and less family-centred values and attitudes are associated with lower fertility. These findings suggest that the negative educationfertility link operates in part via the less traditional values centred on leisure time and self-realisation that are more typical of the women and men with high levels of education.

The next two contributions, by Alessandra Trimarchi and Jan Van Bavel and by Nitzan Peri-Rotem, look at the role of the partner's education in determining an individual's union status at first birth. This research is motivated in part by the ongoing debate about the deepening social status divides in parenthood, and the likely long-term consequences of these differences for families and children. The study by Trimarchi and Van Bavel uses Generation and Gender Surveys (GGS) data for 12 European countries to look at the effects of both partners' education on the pathways to the first birth by analysing separately transitions from cohabitation to marriage, from marriage to first birth, and from cohabitation to first birth. The authors find that the partners' combined education (i.e. the "overall human capital of the couple") has a stronger effect on the likelihood of having a child within marriage than the relative education levels of the partners. Highly educated partners are more likely to get married once they start planning to have a child together, even if they were initially cohabiting. In addition, if at least one partner in a couple has tertiary education, the couple's chances of non-marital childbearing are lower. Peri-Rotem analyses changes in the partnership context at first birth, looking at the influence of education and employment status among women and couples in Great Britain. Using the British Household Panel Survey (BSPS), she focuses on the period between 1991 and 2012, when childbearing in cohabiting unions increased 
across all social groups in Britain, and marriage rates fell among women and men with medium and low levels of education. In line with the findings of Trimarchi and Van Bavel, the results of this study confirm that the educational levels of both partners influence the likelihood of a couple experiencing a non-marital first birth, with the man's (but not the woman's) unemployment or inactivity being conducive to out-of-wedlock childbearing. The educational level of the male partner has the strongest influence on the marital status at first birth of medium educated women. These findings confirm that a couple's economic resources, earning capacity, and level of economic insecurity have large effects on their marital status at first birth.

The following two contributions, by Christos Bagavos and by Anja Oppermann, use census and microcensus data to explore the link between education and cohort fertility, while also explicitly considering childlessness. Bagavos applies a couple perspective to study the influence of women's and men's education on completed fertility, childlessness, parity progression ratios, and first birth timing in Greece. Using three rounds of population census data (1991, 2001, and 2011) to analyse the fertility behaviour of native-born couples born between 1945 and 1969, he finds that there are surprisingly small differences between different educational pairings in final levels of childlessness. At the same time, homogenous couples with low levels of education have considerably higher completed fertility than all the other pairings. This finding may be attributable to the higher second and third birth transition rates of these couples, or to their relatively young ages at the first birth. The negative effect of education on fertility appears to be more pronounced for women. Couples in which the woman is highly educated are found to have comparatively low fertility and a late first birth, especially if the woman is married to a man with less education. This pattern persists across cohorts, and disparities in first birth timing have widened considerably. These results suggest a lack of policy support and continuing low levels of compatibility between career and family plans. Oppermann's study uses German microcensus data from 2008 to look at the links between educational attainment, field of study, childlessness, and completed fertility. Her analysis focuses on women in western Germany born in 1955-59, but she also compares the childlessness levels across broader educational field groups in western Germany and eastern Germany, and - drawing from previous research - in Sweden, Austria, and Greece. This analysis reveals a relatively strong educational gradient in childlessness and completed fertility in western Germany, with educational level and field contributing equally to the variation in completed fertility. As in other countries analysed in the past, women in western Germany who were trained in teaching or childcare are found to have lower levels of childlessness and higher levels of completed fertility than women trained in other professions. Oppermann also highlights the role of self-selection, arguing that "the choice of educational field is an expression of preferences or even personality traits that are independent of institutional context".

Sylvie Dubuc uses pooled Labour Force Survey data for the United Kingdom in 2001-10 to reconstruct the timing of childbearing and fertility levels among immigrants and their children (second generation) originating from four Asian 
countries: Bangladesh, Pakistan, China, and India. Dubuc's analysis reveals consistent patterns across these groups as well as among native women, including a tendency among highly educated women (especially women of Chinese origin) to delay childbearing and have relatively low fertility. A large share of the observed fertility differences between women of different ethnic origins is explained by the differences in educational attainment between these groups. Highly educated women of Indian and especially of Chinese origin are found to be far more likely than the UK average to have low and delayed fertility, or to be childless. In contrast, less educated women from Bangladesh and Pakistan - who make up substantial shares of the immigrant populations from these countries - are shown to have higher fertility than the UK average, with many having three or four children.

The final contribution, by Maria Rita Testa and Fabian Stephany, examines fertility intentions by level of education. The study is the first to use meta-analysis to provide a systematic quantitative assessment of the past research on the topic, assessing 29 published studies covering 13 countries. The results indicate that the gradient is not significant in most study lines, but that several studies show a positive educational gradient in intended family size for women and men. This trend appears to be driven by the positive gradient in second birth intentions among women with one child, which has been explained by the "time squeeze" effect; i.e. the tendency among highly educated women to start childbearing at later ages, and thus to attempt to realise their reproductive plans over a relatively short period of time (Kreyenfeld 2002). The regression model with country clusters reveals that the regional divides in reproductive intentions are somewhat counter-intuitive: a strong positive educational gradient in fertility intentions is reported for Southern Europe, where the educational gradient in fertility is strongly negative.

\section{Will highly educated women have more children in the future?}

This volume of the Vienna Yearbook features six invited debate contributions. The authors were asked to discuss the question of whether highly educated women will have more children in the future. Because the precise question and the time horizon were not explicitly stated, the authors were invited to reflect on the general question of whether the fertility of better educated women is likely to eventually surpass that of their less educated counterparts, thereby reversing the long-term negative association between education and fertility; or whether better educated women are likely to have higher fertility in the future than today. Similarly, the authors were free to consider the general question of whether a future reversal in the educationfertility association is more likely to be caused by a recovery in the fertility of highly educated women, or by a decline in the fertility of less educated women.

The relatively open debate format resulted in engaging contributions that discuss the nature of the education-fertility relationship from different angles. The authors provide a wide range of theoretical and empirical arguments about the past and the likely future of this relationship, and about the forces shaping it. They also shed 
critical light on the prevailing theoretical approaches, especially the rational "new home economics" approach pioneered by Gary Becker (e.g. Becker 1981).

Alícia Adserà argues that due to their rising share in the population, the childbearing behaviour of highly educated women will be crucial for the future development of fertility. In particular, she looks at the extent to which labour marketrelated factors, institutions, and policies facilitate or hinder the realisation of fertility intentions among the better educated, and notes the continuation of the gender wage gap and the "sorting" of women into lower paid and more protected job positions. Wolfgang Lutz highlights the empowering capacity of education, which allows highly educated women and men to plan and "organise their lives according to their intentions"; and, as a result, to "reach their personal target for family size, regardless of what the target is". Understanding the fertility targets of highly educated women is therefore "key for understanding future trends in fertility". He also emphasises that the conflict between pursuing a professional career and having a family is most acute among highly educated women. Similarly, Maria Rita Testa argues that in order to design effective policy interventions, it is important to understand the fertility intentions of women and men with different levels of education. Noting that the two-child family norm is shared by all educational groups across the low-fertility countries, Testa points out that the main challenge faced by highly educated women in meeting the two-child family target is their tendency to delay family formation. She observes that women may find it difficult to have children later in life not only because infertility increases with age, but because they may have competing activities and goals.

Jan Van Bavel puts a spotlight on the role of the male partner in fertility, and stresses the importance of understanding men's preferences, attitudes, and skills regarding family formation. He also looks at the extent to which union formation and assortative mating drives some of the observed education-fertility interactions, noting that highly educated women are increasingly likely to be the main "breadwinner" in the family, and are more likely to partner with a man who is willing to take on more childcare and family responsibilities. With the reversal of the gender gap in education, the Beckerian framework of specialised gender roles is seen as increasingly outdated, and thus less useful in analyses of fertility decisions. Diego Ramiro-Fariñas, Francisco J. Viciana-Fernández and Víctor Montañés Cobo comment on the implications of changes in the educationfertility relationship in the context of the rapidly changing labour market conditions in the region of Andalusia in Spain, especially during the recent economic crisis. Remarkably, they point out, the fertility rates among highly educated women were the least affected during the economic downturn. They observe that over this period, employment status was of key importance: women with a permanent job continued to have relatively high first and second birth rates regardless of their educational level, whereas fertility plummeted among non-working women with tertiary as well as lower levels education. Finally, Gøsta Esping-Andersen focuses on the role of the "gender revolution" and of more gender-symmetric practices in allowing better educated women to achieve a larger family size. He argues that a "return to fertility 
levels that are more aligned with people's preferences will require the consolidation of a new, 'gender-egalitarian' family equilibrium". According to Esping-Andersen, this trend can be encouraged by institutional adaptations, including improvements in reconciliation policies.

Do these contributions offer an explicit answer to the question posed in the debate? Most authors suggest that the answer is "it depends". Notably, the future fertility of better educated women will depend on their fertility goals and broader institutional conditions. Most of the authors envision a broad convergence in fertility among women with different levels of education - in line with their fertility preferences - rather than clear reversals in the education-fertility gradients (e.g. Adserà, Lutz). What then are the conditions that will determine the future educationfertility link? Lutz suggests that the fertility goals of highly educated women will be of key importance. In contrast, Van Bavel emphasises the role of men's preferences and plans. Adserà stresses the role of labour market policies in reducing the barriers to fertility among the better educated. Similarly, Ramiro-Fariñas et al. highlight the role of labour market opportunities, especially in reducing unemployment and temporary employment. Testa emphasises the reconciliation of work and family life and the promotion of gender equality in the family and in the labour market. EspingAndersen focuses on the role of gender egalitarianism, while stating most clearly the expectation that highly educated women are likely to have more children in the future. In contrast, Ramiro-Fariñas et al. suggest that unstable labour market conditions will not "give much potential for an increase in fertility in any of the education groups".

\section{Discussion: the continuing diversity of educational gradients in fertility across countries}

The debate contributions and research articles in this volume provide a broad range of perspectives on the changing nature of the education-fertility relationship in different contexts and populations, and its future development. For the analysed periods and cohorts of women and men (typically, cohorts born up to the late 1960s), these studies show a continuation of the negative educational gradient in fertility among women, and a weaker and less significant gradient among men. Among couples and among women in particular, having a higher level of education is still associated with lower fertility. The size of the educational gradient differs by welfare regime, and appears to have weakened in the more recent cohorts in a range of countries. It is, however, unclear whether the recently observed flattening of the educational gradient in fertility in the Nordic countries and Belgium (e.g. Kravdal and Rindfuss 2008; Neels 2012; Jalovaara et al. 2017), as well as in the United States (e.g. review contribution by Adserà in this volume), signals a wider trend across the developed countries. Additional empirical evidence drawn from the Cohort Fertility and Education (CFE) database suggests that there is considerable 
cross-country variation among women born between 1940 and 1970, with women in some countries (e.g. Finland) experiencing a convergence across educational groups to a stable moderately low fertility level, and women in other countries (e.g. Korea) experiencing a convergence across educational groups to a very low fertility level. Moreover, women in a number of countries (e.g. Spain) seem to be following a "permanent difference model" in which different groups have similar relative fertility levels over time, while women in a few other countries (e.g. Serbia) are experiencing a widening of education-fertility differentials (see Figure 1).

Most of the contributors to this volume appear to be reluctant to offer any clear predictions of future trends. However, the authors seem to share the view that a series of interrelated factors will likely affect the ability of better educated women and men to realise their reproductive plans, and thus to have larger families. These factors include the greater stability of unions and marriages among higher educated women and men, the widening disparities in income and labour market prospects, the spread of gender-egalitarian attitudes and the actual shift towards a more equal division of childcare and household tasks among higher educated couples, and the expansion of work-family reconciliation policies. Some of these factors might reinforce each other: if highly educated women and men increasingly prefer to live with similarly highly educated partners, these "power couples" (Dribe and Stanfors 2010) might become even more advantaged than they are currently. While highly educated people are likely to have the wealth, career success, and social capital needed to realise their reproductive plans, less educated people are likely to face economic insecurity, a scarcity of jobs, unstable partnerships, and unstable family contexts at first birth that will make it harder for them to reach their fertility goals. It is possible that women and especially men in economically disadvantaged positions will increasingly "miss out" on their reproductive plans, and will have higher rates of childlessness than the other social groups. In addition, as discussed in the contributions by Adserà, Trimarchi and Van Bavel, and PeriRotem in this volume, depleted parental resources might affect the well-being of children, resulting in lifelong disadvantages for people born into these families.

The functioning of the labour market and gender equality are two context-specific factors that are repeatedly mentioned in the contributions. These two factors may also have the greatest impact on the future fertility of highly educated women, men, and couples; as well as on overall fertility trends. Considerations such as whether the person has a relatively secure job, a stable income, and sufficient socioeconomic resources are likely to be even more salient for fertility decisions in the future than they were in the past. Therefore, especially among young adults, factors such as unemployment, job instability, work flexibility, and career prospects may be expected to have large effects on the future educational gradient in fertility, as Adserà argues in her review. Gender inequalities in the division of childcare and household tasks still hinder the fertility decisions of many university educated women. Gender inequalities also persist in the labour market, where these differences may paradoxically support the fertility decisions of women and their partners. Women's and men's jobs continue to be segregated to a large degree. Even 
Figure 1:

Educational differences in completed fertility among women born in 1940-1970
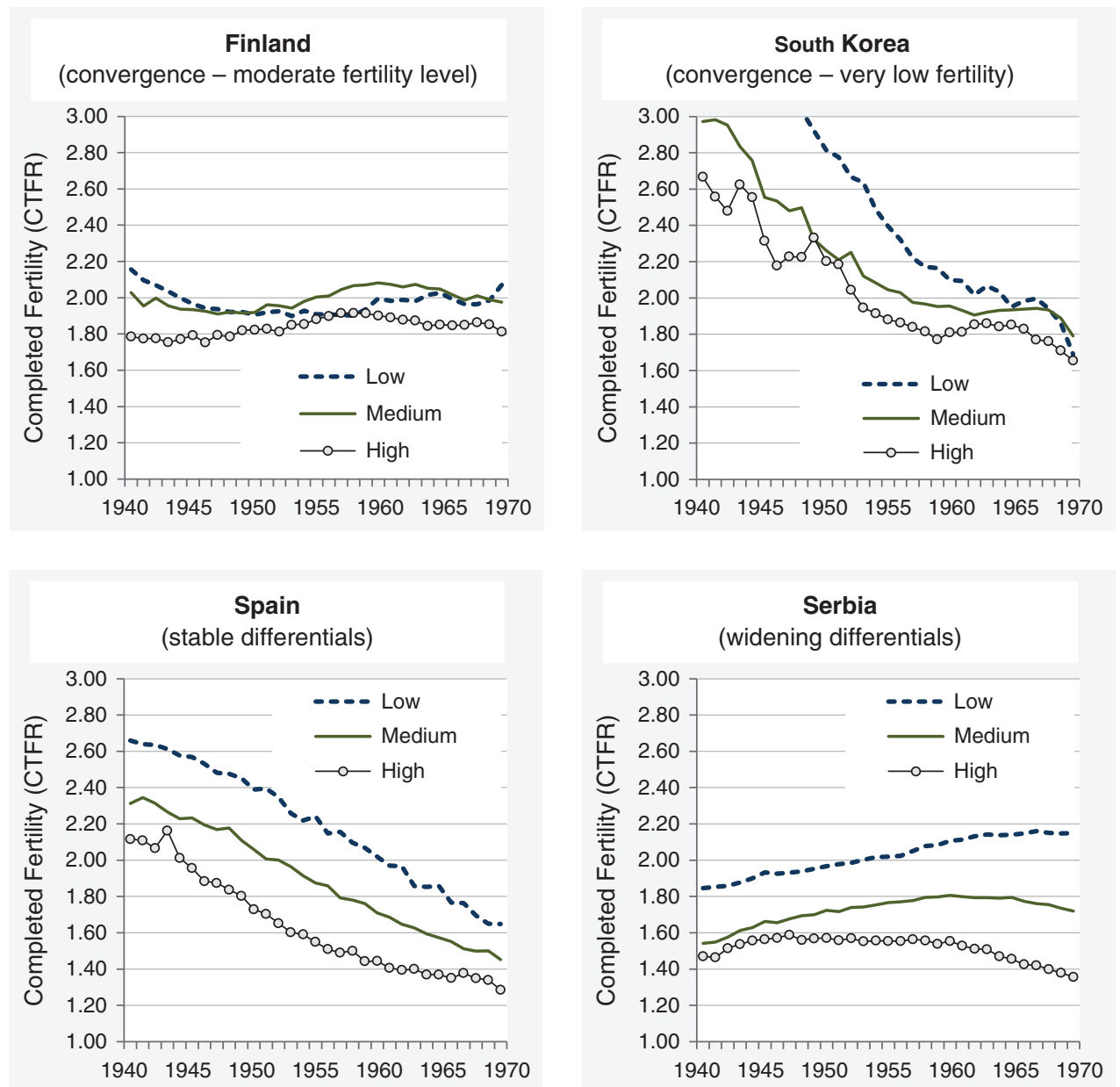

Notes: Educational categories are defined using standardised 1997 ISCED categories: Low: ISCED 0-2 (up to lower secondary and the second stage of basic education); Medium: ISCED $3+4$ (upper secondary and short post-secondary education); High: ISCED $5+6$ (tertiary education, university degree). For details on country-specific categories and educational systems, see the country documentation in the CFE database. Data for Finland and Spain pertain to native-born women only and exclude migrant women.

Sources: Data provided in the Cohort Fertility and Education (CFE) database (www.cfe-database.org), accessed on 18 December 2017. The original data are based on 2015 register data for Finland (provided by Statistics Finland), 2010 census data for South Korea (1\% sample), 2011 census data for Spain, and 2011 census data for Serbia. 
in the highly egalitarian Nordic countries, women are overrepresented in health care, social work, education, and retail jobs that are more compatible with raising children (OECD 2017b).

Despite the vast literature on education and fertility, the discussions in this volume clearly show that many aspects of this relationship are not sufficiently covered. In conclusion, we provide a selected list of themes that should be explored in future research.

- Focus on more detailed and more precisely defined educational categories. Whereas in the past the majority of people had low levels of education, today in some countries the majority of the younger population have tertiary education. Thus, research would benefit from a wider application of a standardised framework that allows for a comparison of educational groups over time through, for example, the use of relative rather than absolute educational categories. A more refined system of categorisation is also needed to study the fertility of women and men at the top of the education distribution.

- Spotlight on values and competing preferences. As Lakomý's article in this volume shows, religiosity, values, and lifestyle preferences are important predictors of fertility behaviour. Future research should take these factors into account. In cross-sectional surveys individual's values and preferences are expressed at the time of survey, i.e. after the analysed life course event has taken place. As a result, this type of analysis may be biased by ex-post causal attribution (Hoem and Kreyenfeld 2006). The use of panel data can help researchers avoid these a posteriori adjustments, although attrition can introduce other types of biases.

- Spotlight on men. Much of the research in this volume has included the perspectives of men and of couples. Still, as Van Bavel points out in the debate section, more studies are needed to shed light on men's values and plans, and on their family history and fertility levels.

- Research on subgroups and minorities. Migrants and other population groups often face different constraints and opportunities than the majority population. Moreover, as they are socialised in a different context, they tend to have distinct values and preferences. As Dubuc's research in this volume shows, differences in the educational attainment levels of migrant groups may account for a substantial portion of their differences in completed fertility. Adserà points out that migrants are likely to continue to influence fertility in the receiving countries, and that their selectivity in terms of their region of origin, skills, and educational levels will be important in determining the future course of their fertility, and whether it converges with the fertility of the majority population.

- Life course perspective on partnership, family formation, and reproduction. Highly educated women and men tend to start the family formation process later than their less educated counterparts. While this is partly because these individuals are older when they complete their education, they also tend to 
wait a long time after their graduation before having a first child (e.g. Neels et al. 2017). Thus, highly educated women and couples face a high risk of infertility, which could in turn lead to involuntary childlessness or having a smaller family than intended (as is pointed out by Testa in the debate section). More studies on the intersection of life course trajectories, first birth timing, ultimate family size, and age-related infertility are needed to better understand the link between late family formation and ultimate fertility among the highly educated.

- Joint effects of education and family preferences. Further exploration of the purposeful sequencing of births and educational outcomes in women's, men's, and couples' lives is called for; as is further investigation of the joint effect of preferences for both educational outcomes and family formation.

- Future of work and digital technologies. Finally, more research is needed on the likely implications of the rapidly changing nature of technology, employment, and labour relations for the fertility patterns of different education groups. Adserà's review article in this volume shows that these topics are also of key importance when considering the likely shift in educational gradients in fertility in the future.

\section{Acknowledgements}

Work by T. Sobotka and É. Beaujouan on this issue of the Vienna Yearbook of Population Research was partly funded by the European Research Council under the European Union's Seventh Framework Programme (FP7/2007-2013)/ERC Grant agreement No. 284238 (EURREP Project). Work by J. Van Bavel was partly supported by the European Research Council under the European Union's Seventh Framework Programme (FP/2007-2013)/ERC Grant Agreement no. 312290 (GENDERBALL Project). The authors thank Natalie Nitsche for her comments and suggestions on the earlier drafts of this text and Miriam Hils for thorough copy editing.

\section{References}

Becker, G. S. 1981. Treatise on the Family. Cambridge: Harvard University Press.

Cleland, J. 2009. Education and future fertility trends, with special reference to midtransitional countries. Completing the fertility transition, United Nations Population Bulletin, Special Issue Nos. 48/49, 2002 (2009).

Dribe, M. and M. Stanfors 2010. Family life in power couples. Continued childbearing and union stability among the educational elite in Sweden, 1991-2005. Demographic Research 23: 847-878.

Esping-Andersen, G. and F. C. Billari 2015. Re-theorizing family demographics. Population and Development Review 41(1): 1-31. 
Goldscheider, F., E. Bernhardt and T. Lappegård 2015. The gender revolution: A framework for understanding changing family and demographic behavior. Population and Development Review 41(2): 207-239.

Hoem, J. M. and M. Kreyenfeld 2006. Anticipatory analysis and its alternatives in life-course research: Part 1: Education and first childbearing. Demographic Research 15: 461-484.

Jalovaara, M., G. Neyer, G. Andersson, J. Dahlberg, L. Dommermuth, P. Fallesen and T. Lappegård 2017. Education, gender, and cohort fertility in the Nordic countries. Stockholm Research Reports in Demography 2017: 6.

James, K. S., V. Skirbekk and J. Van Bavel 2012. Education and the global fertility transitionForeword. Vienna Yearbook of Population Research 2012(10): 1-8.

Klesment, M. and J. Van Bavel 2017. The reversal of the gender gap in education, motherhood, and women as main earners in Europe. European Sociological Review 33(3): 465-481.

Kravdal, $\varnothing$. and R. R. Rindfuss 2008. Changing relationships between education and fertility: a study of women and men born 1940 to 1964. American Sociological Review 73: 854-873.

Kreyenfeld, M. 2002. Time-squeeze, partner effect or self-selection? An investigation into the positive effect of women's education on second birth risks in West Germany. Demographic Research 7(2): 15-48.

Martín-García, T. 2008. A reassessment of the role of women's education in existing fertility research. Genus 64(1-2): 131-157.

McLanahan, S. and C. Percheski 2008. Family structure and the reproduction of inequalities. Annual Review of Sociology 34: 257-276.

Mills, M. and H. P. Blossfeld 2013. The Second Demographic Transition meets globalization: A comprehensive theory to understand changes in family formation in an era of rising uncertainty. Chapter 1 in Negotiating the life course. Stability and change in life pathways, ed. A. Evans and J. Baxter, 9-33. Springer: Netherlands.

Mills, M., R. R. Rindfuss, P. McDonald and E. te Velde 2011. Why do people postpone parenthood? Reasons and social policy incentives. Human Reproduction Update 17(6): 848-860.

Neels, K., M. Murphy, M. Ní Bhrolcháin and É. Beaujouan 2017. Rising educational participation and the trend to later childbearing. Population and Development Review 43(4): 667-93.

Ní Bhrolcháin, M. and É. Beaujouan 2012. Fertility postponement is largely due to rising educational enrolment. Population Studies 66(3): 311-327.

Nisén, J. 2016. Education and fertility: A study on patterns and mechanisms among men and women in Finland. Doctoral Thesis. University of Helsinki. Publications of the Faculty of Social Sciences 31-2016.

OECD. 2014. Society at a Glance 2014. The crisis and its aftermath. OECD Publishing. Available at http://www.oecd.org/els/societyataglance.htm.

OECD. 2016. Society at a Glance 2016. A spotlight on youth. Paris: OECD Publishing. Available at OECD Publishing, Paris. DOI: 10.1787/9789264261488-en.

OECD. 2017a. Education attainment statistics. OECD online library, data accessed 13 December 2017; DOI: 10.1787/025421e5-en. 
OECD. 2017b. The pursuit of gender equality: An uphill battle. Paris: OECD Publishing. DOI: $10.1787 / 9789264281318$-en.

Perelli-Harris, B. and M. Lyons-Amos 2016. Partnership patterns in the United States and across Europe: The role of education and country context. Social Forces 95(1): 251-282.

Piketty, T. and G. Zucman 2014. Capital is back: Wealth-income ratios in rich countries 1700-2010. The Quarterly Journal of Economics 129(3): 1255-1310.

Stange, K. 2011. A longitudinal analysis of the relationship between fertility timing and schooling. Demography 48(3): 931-956.

Van Bavel, J. 2012. The reversal of gender inequality in education, union formation and fertility in Europe. Vienna Yearbook of Population Research 2012(10): 127-154.

Van Bavel, J. and M. Klesment 2017. Educational pairings, motherhood, and women's relative earnings in Europe. Demography 54(6): 2331-2349.

Webbink, D., N. G. Martin and P. M. Visscher 2011. Does teenage childbearing reduce investment in human capital? Journal of Population Economics 24(2): 701-730. 
DEMOGRAPHIC DEBATE 
